\title{
FATORES ASSOCIADOS AO ISOLAMENTO SOCIAL E SOLIDÃO ENTRE IDOSOS COMUNITÁRIOS EM TEMPOS DE PANDEMIA
}

Caroline de Fátima Ribeiro Silva; Universidade Federal do Amapá; carolribeiro_30@hotmail.com Juliana de Souza da Silva; Universidade Federal do Amapá; julianasouza_silva22@hotmail.com Fabrícia Coelho de Araújo; Universidade Federal do Amapá; fabricia_28@live.com Daniela Gonçalves Ohara; Universidade Federal do Amapá; dani_ohara@hotmail.com Areolino Pena Matos; Universidade Federal do Amapá; areolino.matos@gmail.com Ana Carolina Pereira Nunes Pinto; Universidade Federal do Amapá; anacarolinapnp@hotmail.com Maycon Sousa Pegorari; Universidade Federal do Amapá; mayconpegorari@yahoo.com.br

\section{RESUMO}

Introdução: Embora esforços tenham sido dispendidos na tentativa de encontrar tratamentos e vacinas eficazes para combater a COVID-19, outras demandas como a abordagem aos aspectos psicossociais precisam ser consideradas e nenhum estudo anterior investigou os fatores associados ao isolamento social e solidão entre idosos na pandemia atual. Objetivo: Analisar a associação entre o isolamento social e a solidão com as características socioeconômicas e de saúde e as variáveis relacionadas à COVID-19 em idosos comunitários em tempos de pandemia. Métodos: Estudo transversal conduzido com 86 idosos $(71,78 \pm 6,98$ anos) contatados via inquérito telefônico em Macapá/AP. Utilizou-se formulário estruturado para as variáveis socioeconômicas, clínicas e de saúde, isolamento social e solidão, e as relacionadas à COVID-19. Procedeu-se a estatística descritiva e inferencial pelo teste de correlação de Pearson e modelo de regressão linear. Resultados: 9,3\% informaram diagnóstico positivo para covid-19, e desses, 3,5\% foram hospitalizados. Referiram sentimentos de solidão $20,9 \%$ e 23,3\% se encontravam socialmente isolados. As médias

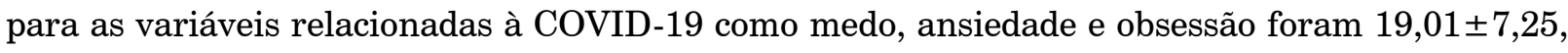
$1,01 \pm 1,90$ e 2,84 $\pm 3,28$, respectivamente. Houve correlação positiva e moderada entre as variáveis solidão e número de doenças; e fraca entre as variáveis solidão e número de medicamentos, sintomas depressivos e risco para sarcopenia. $\mathrm{O}$ modelo de regressão linear indicou que o maior escore de solidão associou-se ao maior número de doenças $(\beta=0,288 ; p=0,007)$. Conclusão: Os resultados deste estudo sugerem uma provável resiliência dos idosos frente à COVID-19, apesar da associação da variável solidão com o número de doenças em tempos de pandemia.

Palavras-chave: COVID-19; Idoso; Isolamento social; Solidão. 